\section{PEADdonto Revista Eletrônica Acervo Odontológico}

\section{RELATO DE CASO}

Recebido em: 3/2020

Aceito em: 3/2020

Publicado em: 4/2020

\title{
Vedamento de comunicação buco-sinusal com prótese obturadora palatina: relato de caso
}

\author{
Buco-sinusal communication fence with palatine shutter prosthesis: case report
}

Sellado de la comunicación buco-sinusal con prótesis de llenado palatino: reporte de caso

Rita de Cássia Araújo Fonseca ${ }^{1}$, Franklin Barbosa da Silva ${ }^{2 *}$.

\begin{abstract}
Resumo: O objetivo deste estudo é relatar um caso de reabilitação com a confecção de uma prótese obturadora palatina, feita após remoção cirúrgica de carcinoma mucoepidermóide localizado na região do palato que ocasionou uma comunicação buco-sinusal. Paciente com 52 anos de idade, gênero masculino, procurou atendimento na clínica de prótese da Universidade Nilton Lins relatando ter passado por cirurgia para remoção de carcinoma mucoepidermóide, e, que apresentou dificuldades na fonação, deglutição e mastigação após o procedimento. Para solução do caso optou-se pela confecção de uma prótese obturadora palatina. Iniciou-se pela moldagem de estudo com alginato, a seguir o modelo resultante foi levado ao delineador para planejamento da estrutura metálica do obturador. Planejou-se uma prótese removível com conector maior barra dupla, quatro grampos geminados para retenção e porção obturadora em acrílico sobre sela posterior. Após isso, fez-se a confecção dos nichos seguida da moldagem de trabalho. O modelo foi encaminhado ao laboratório e na consulta seguinte a prótese foi adaptada com sucesso em boca. Com a instalação observou-se melhora nas funções orais do paciente, diminuindo as consequências da sequela cirúrgica.
\end{abstract}

Palavras-chave: Carcinoma mucoepidermóide, Prótese maxilofacial, Obturadores palatinos.

\begin{abstract}
The objective of this study is to report a case of rehabilitation with the manufacture of a palatal obturator prosthesis, made after surgical removal of mucoepidermoid carcinoma located in the region of the palate that caused a bucco-sinusal communication. A 52-year-old male patient came at the prosthesis clinic of Nilton Lins University, reporting having undergone surgery to remove mucoepidermoid carcinoma, and who had difficulties in phonation, swallowing and chewing after the procedure. To solve the case, it was decided to make a palatal obturator prosthesis. It started by study molding with alginate, then the resulting model was taken to the paralelometer for planning the metal structure of the obturator. A cast partial denture with double bar connector, four twinned clasps for retention and an acrylic obturator portion on a posterior saddle was planned. After that, the niches were made followed by work molding. The model was sent to the laboratory and at the next consultation the prosthesis was successfully adapted in the mouth. With the installation, an improvement in the patient's oral functions was observed, reducing the consequences of the surgical sequel.
\end{abstract}

Keywords: Carcinoma mucoepidermoid, Maxillofacial prosthesis, Palatal obturators.

1 Universidade Nilton Lins, Manaus - AM. *E-mail: frank_barbosa@hotmail.com 
Resumen: El objetivo de este estudio es informar un caso de rehabilitación con la fabricación de una prótesis de relleno palatino, realizada después de la extirpación quirúrgica del carcinoma mucoepidermoide ubicado en la región del paladar que causó una comunicación buco-sinusal. Un paciente masculino de 52 años buscó atención en la clínica de prótesis de la Universidad de Nilton Lins, informando que se sometió a una cirugía para extirpar el carcinoma mucoepidermoide y que tuvo dificultades para fonación, deglución y masticación después del procedimiento. Para resolver el caso, se decidió hacer una prótesis de relleno palatino. Comenzó moldeando el estudio con alginato, luego el modelo resultante fue llevado al delineador para planificar la estructura metálica del obturador. Se planeó una prótesis removible con un conector de barra doble más grande, cuatro abrazaderas gemelas para retención y una porción de relleno acrílico en una silla posterior. Después de eso, se hicieron los nichos seguidos de moldeo de trabajo. El modelo fue enviado al laboratorio y en la siguiente consulta la prótesis se adaptó con éxito en la boca. Con la instalación, se observó una mejora en las funciones orales del paciente, reduciendo las consecuencias de la secuela quirúrgica.

Palabras clave: Carcinoma mucoepidermoide, Prótesis maxilofacial, Obturadores palatinos.

\section{INTRODUÇÃO}

O câncer da cavidade oral ocupa a 15 $5^{\text {a }}$ posição entre todos os cânceres em nível mundial, sendo que destes, mais da metade estão entre os homens (198.975 casos novos). Segundo as informações do Globocan/larc, em 2012, foram estimados 300.373 casos novos de cânceres de lábio e cavidade oral no mundo. Em relação à mortalidade, foi responsável por 145.353 óbitos em 2012 (IARC, 2012). No Brasil, está entre os 10 tipos de cânceres mais incidentes no sexo masculino, ocupando a $5^{a}$ posição no ranking. Para os anos de 2018 e 2019 foram estimados 11.200 casos novos em homens e 3.500 em mulheres. Na região Norte foram estimados 330 novos casos em homens e 170 em mulheres, e, em Manaus, 80 novos casos em homens e 30 em mulheres (INCA, 2018).

Os cânceres da cavidade oral são classificados em dois grupos, o carcinoma de células escamosas que corresponde à grande maioria dos casos e o carcinoma de glândulas salivares. Este último pode ser classificado em carcinoma mucoepidermóide, carcinoma adenoide cístico e adenocarcinoma. O carcinoma mucoepidermóide é o tumor maligno das glândulas salivares mais frequentemente encontrado na cavidade oral. Em mais de metade dos casos, esse tumor está associado a uma translocação cromossômica balanceada (11;19) (q21;p13), que gera um gene fusionado composto por porções dos genes MECT1 e MAML2as (LINGEN MW, 2016; MATHEW AL, et al., 2017).

Os principais fatores associados a este tipo de neoplasias são o tabagismo, etilismo e exposição solar ultravioleta. Outros fatores descritos na literatura que também contribuem para a carcinogênese são a infeção pelo papilomavírus humano (HPV), dieta pobre em frutas e vegetais e má higiene bucal (ACC, 2005). O tratamento depende do estágio clínico, localização e grau histopatológico, podendo ser com radioterapia, quimioterapia ou cirurgia que é a primeira escolha de tratamento do câncer oral (ARUN KUMAR VR, et al., 2016). Um diagnóstico precoce é de grande importância para que não ocorra perdas teciduais extensas no caso da remoção cirúrgica (ESTEVES AJ, et al., 2017)

O tratamento cirúrgico de neoplasias malignas localizadas no palato ocasiona uma comunicação bucosinusal, acarretando transtornos funcionais na fonação, deglutição e mastigação, prejudicando a qualidade de vida do paciente (SHIBAYAMA R, et al., 2016; DOS SANTOS DM, et al., 2016). O tratamento reabilitador de primeira escolha são as cirurgias plásticas reconstrutoras, porém quando não é possível devido ao estado de saúde do paciente, a extensão e gravidade da comunicação, uma alternativa viável é a confecção de próteses obturadoras, que se tratam de peças protéticas com o intuito de separar artificialmente a cavidade bucal da nasal (OTTRIA L, et al., 2014; CHEN C, et al., 2016). Próteses obturadoras provisórias são utilizadas logo após a cirurgia para ajudar na cicatrização e diminuir o risco de inflamações e infecções (ARUN KUMAR VR, et al., 2016; SINGH M, et al., 2013). No caso de cura total faz-se prótese definitiva, porém levando em conta o quadro específico de cada paciente é necessário um acompanhamento caso ele venha a ter uma recidiva (LINGEN MW, 2016).

REAOdonto | Vol. 1 | e3117| DOI: https://doi.org/10.25248/REAOdonto.e3117.2020 Página 2 de 8 
Além da prótese obturadora convencional que utiliza vários grampos como componentes de retenção, existe uma variedade de obturadores, todas visam recuperar e restabelecer a estética e as funções orais do paciente (ARUN KUMAR VR, et al., 2016). O tratamento com próteses obturadoras pode vir a ser um desafio, o tamanho da comunicação e das estruturas anatômicas que restam para apoiar a prótese podem comprometer a retenção e estabilidade da mesma (KAR S e TRIPATHI A, 2015). O ideal é que o planejamento reabilitador seja aliado a um tratamento multidisciplinar, facilitando a devolução da qualidade de vida e a saúde psicológica perdidas devido ao trauma causado ao paciente (DOMINGUES JM, et al., 2016; RABELO DP, et al., 2018).

Sendo assim, o objetivo deste estudo é relatar um caso de reabilitação com prótese obturadora palatina, após remoção cirúrgica de carcinoma mucoepidermóide localizado na região do palato que ocasionou uma comunicação buco-sinusal.

\section{RELATO DE CASO}

Paciente de 52 anos, gênero masculino, compareceu a clínica de prótese da Universidade Nilton Lins, relatando dificuldade na fonação, deglutição e até mesmo na mastigação. Fazia uso de um obturador palatino de acrílico, no qual não estava satisfeito. Esses fatores estavam afetando sua qualidade de vida. Após exame clínico foi observado que o paciente não possuía ausências de dentes no arco superior, porém com a presença de uma comunicação buco-sinusal na região próxima à fronteira palato duro com palato mole, sendo esta mutilação resultante de uma cirurgia para remoção de carcinoma mucoepidermóide (Figura 1).

Como plano de tratamento optou-se pela confecção de uma prótese obturadora palatina. Inicialmente foi feito o tratamento periodontal, com consecutivas sessões de raspagem para melhorar a higiene e a efetividade das etapas do tratamento. No atendimento realizou-se a moldagem anatômica para obter o modelo de estudo. Utilizando moldeira de alumínio perfurada superior para dentados, alginato (Jeltrate Plus, Dentsply, Brasil) e vazamento com gesso (Durone IV, Dentsply, Brasil) (Figura 2).

Figura 1 - Aspecto inicial intrabucal onde se observa comunicação buco-sinusal.

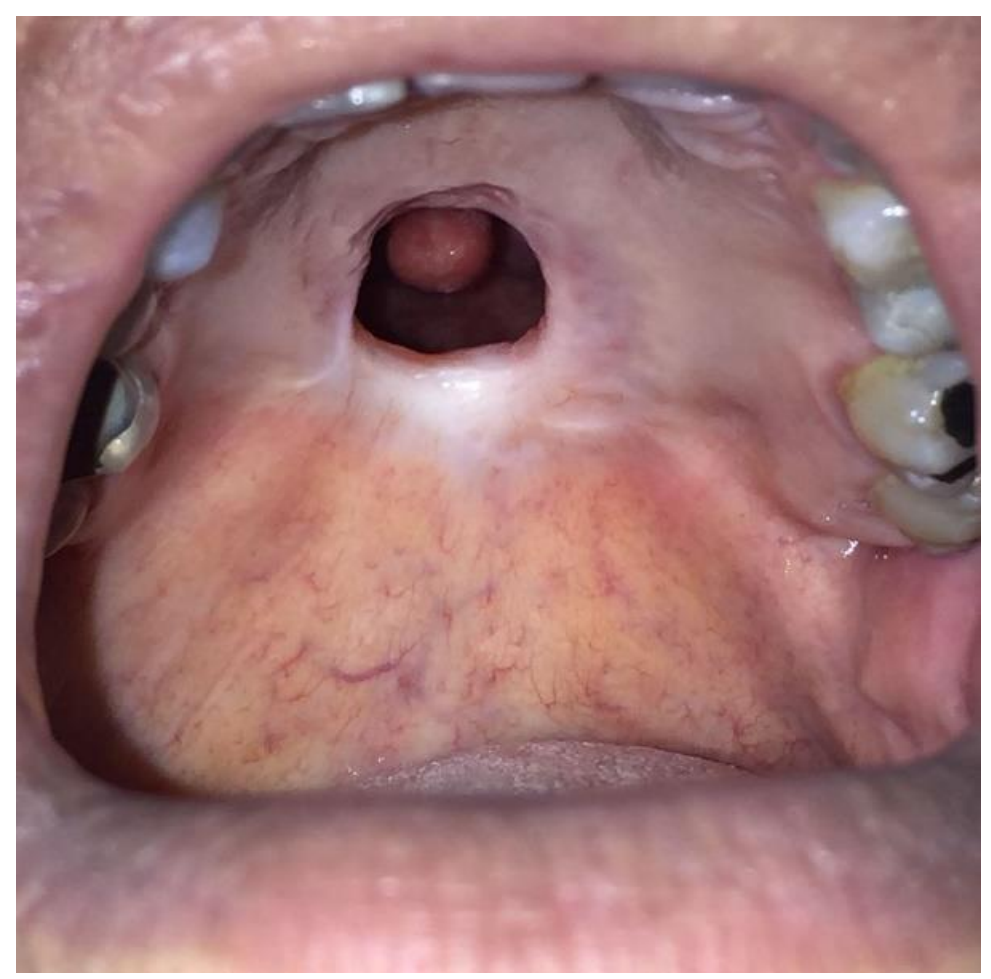

Fonte: Fonseca RCA e Silva FB, 2020. 
Figura 2 - Modelo de estudo.

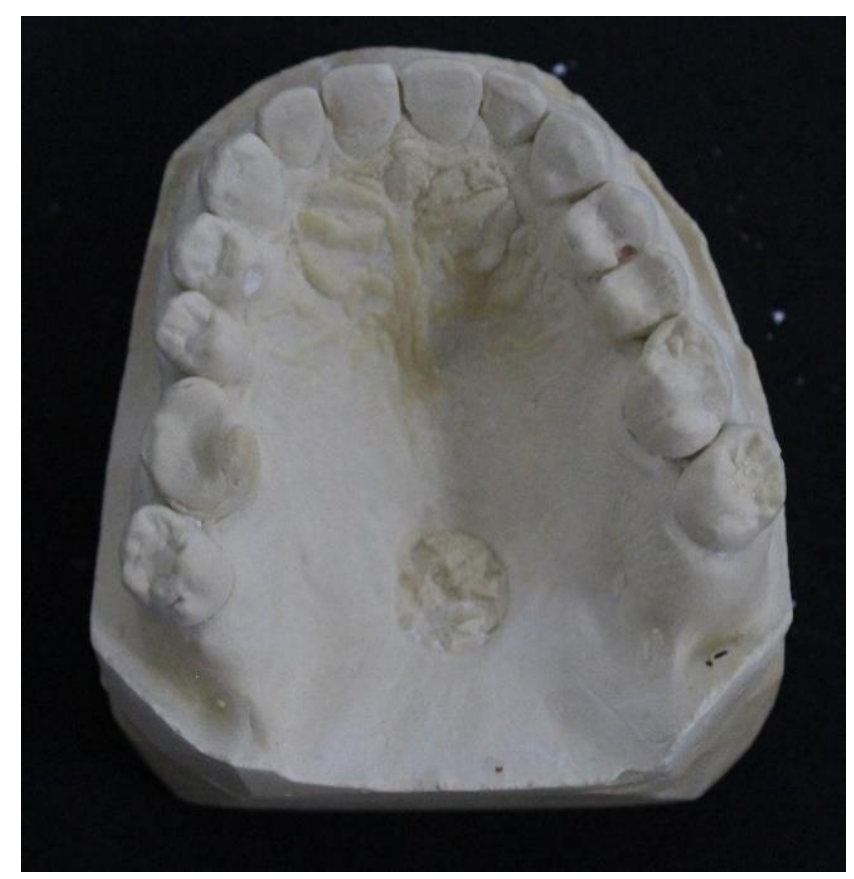

Fonte: Fonseca RCA e Silva FB, 2020.

O modelo foi levado ao delineador para diagnosticar e guiar os preparos em boca. Iniciou-se pela determinação do eixo de inserção e remoção pela ponta analisadora com a associação das duas técnicas: dos três pontos e de conveniência. Logo depois foi marcado o equador protético com a ponta grafite, determinando assim o grau de retenção de cada elemento dentário, por último foi utilizada a ponta calibradora 0,5 para calibrar a quantidade de área retentiva (Figura 3).

Os elementos dentais selecionados como pilares foram 14, 15, 16, 17, 24, 25, 26 e 27 . O conector maior de escolha foi a barra palatina dupla, semelhante uma prótese parcial removível com retenção e distribuição ampla da carga funcional. Os grampos planejados foram os geminados. Foi feita a confecção dos preparos dos nichos com desgastes para apoio oclusal nos dentes pilares utilizando broca diamantada esférica \#1014 em alta rotação sob refrigeração (Figura 4).

Figura 3 - Delineamento com ponta grafite.

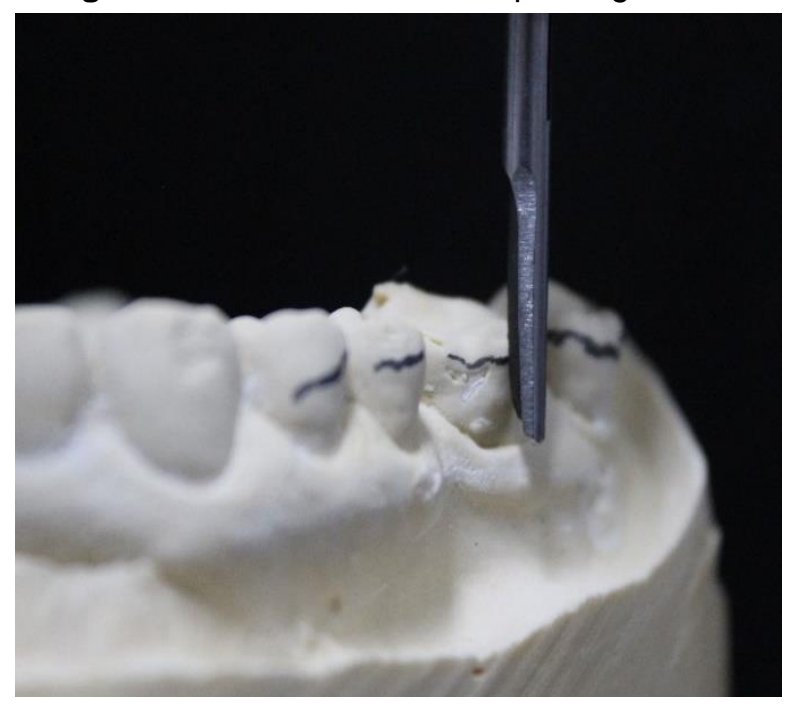

Fonte: Fonseca RCA e Silva FB, 2020. 
Figura 4 - Nichos confeccionados.

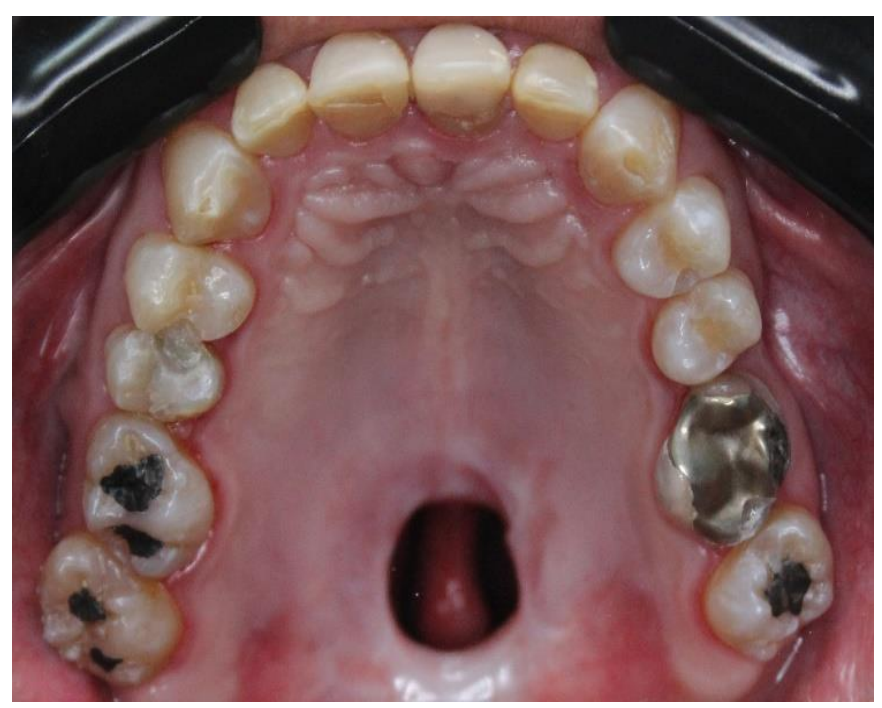

Fonte: Fonseca RCA e Silva FB, 2020.

Após o planejamento no modelo de estudo, obteve-se o modelo de trabalho. Foi realizada a técnica de moldagem dupla utilizando silicone de condensação (Precise SX, Dentsply, Brasil) junto com o uso de filme de PVC. Após a cópia com material denso, realizou-se a moldagem com silicone fluido (Precise SX, Dentsply, Brasil) para obter detalhes das estruturas dentais (Figura 5). Foi feito o vazamento do molde com gesso especial (Durone IV, Dentsply, Brasil), obtendo assim o modelo de trabalho, sendo este enviado para o laboratório para confecção da armação metálica e o vedamento da comunicação com porção de resina acrílica termopolimerizável na sela.

Durante a instalação da prótese foi observada adequada retenção e que faltava um pouco de vedamento da comunicação, sendo necessário realizar reembasamento com resina acrílica autopolimerizável (Figura 6). As orientações de manutenção, higienização e adaptação da prótese foram dadas ao paciente. No retorno após uma semana confirmou-se o sucesso na adaptação e acomodação da prótese.

Figura 5 - Molde de trabalho.

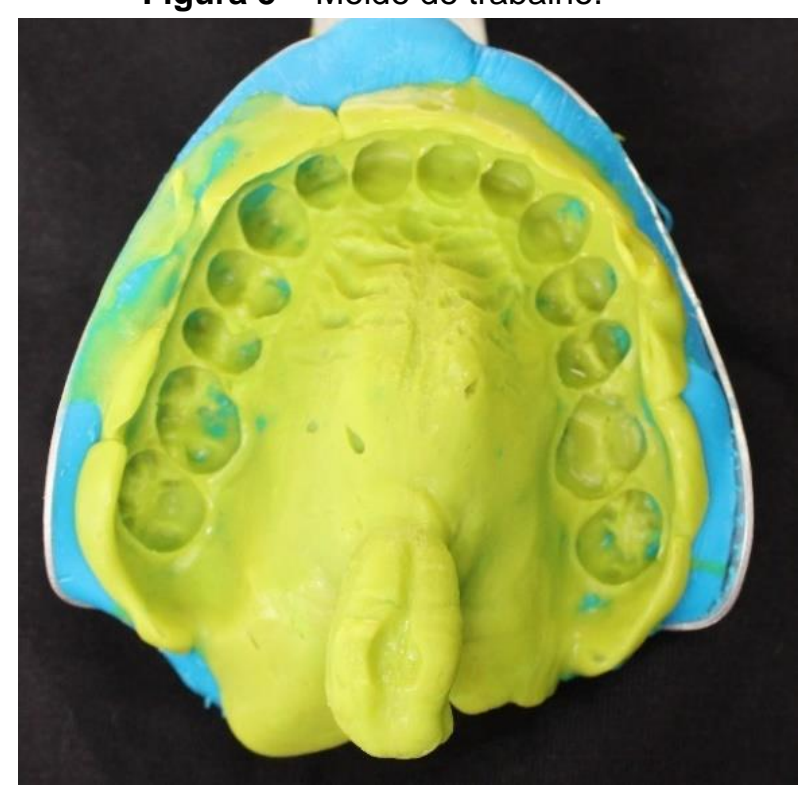

Fonte: Fonseca RCA e Silva FB, 2020. 
Figura 6 - Instalação da prótese.

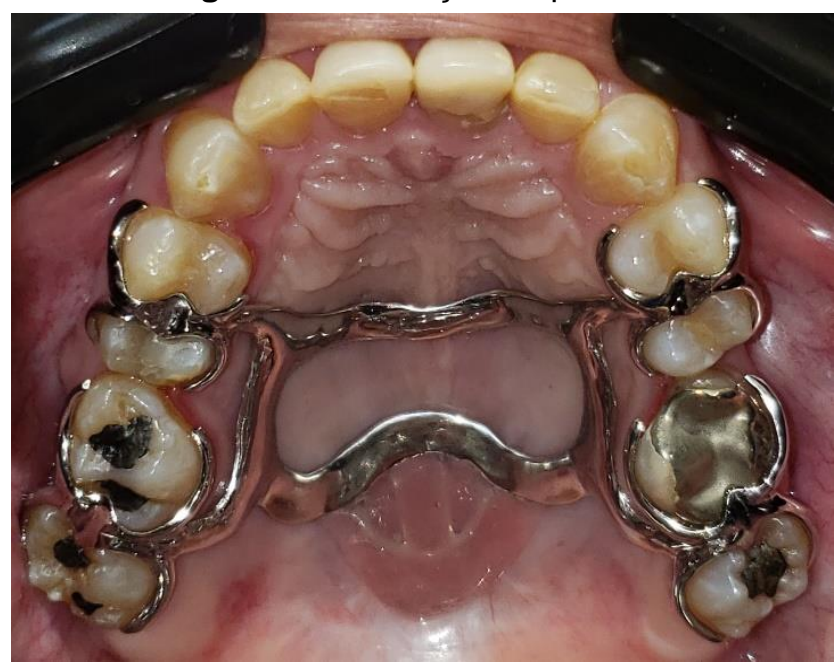

Fonte: Fonseca RCA e Silva FB, 2020.

\section{DISCUSSÃO}

O tratamento de eleição para tumores no palato é a maxilectomia. As mutilações resultantes das cirurgias oncológicas ocasionam grandes deformidades na cavidade oral e até mesmo na face, prejudicando assim a parte funcional, estética e psicológica, em que os não reabilitados apresentam dificuldades na mastigação, deglutição e fonação, além de refluxo alimentar pela cavidade nasal (DOMINGUES JM, et al., 2016; DOS SANTOS DM, et al., 2016). O paciente do caso clínico em questão apresentava-se com uma comunicação buco-sinusal na região de palato mole e dificuldades funcionais após a intervenção cirúrgica, sendo o mesmo considerado como curado, o torna indicado para procedimento reabilitador definitivo que minimizasse o defeito adquirido.

O tratamento para vedamento de comunicação buco-sinusal pode se dá em três fases: protética provisória, cirúrgica e reabilitadora, conforme o caso específico de cada paciente (RABELO DP, et al., 2018). Shibayama $\mathrm{R}$, et al. (2016) afirma que as cirurgias plásticas reabilitadoras em alguns casos são difíceis de serem realizadas, levando em consideração que podem esconder casos de recidivas tumorais em estágio inicial. Segundo Goiato MC, et al. (2016) quando a reabilitação cirúrgica apresenta contraindicações devido as recidivas, anatomia, distúrbios funcionais ou sociais, ou até mesmo quando o paciente não está disposto a mais uma cirurgia, a prótese obturadora torna-se o tratamento de primeira escolha, vedando a comunicação e permitindo a melhora na mastigação, deglutição e fonação.

Próteses obturadoras provisórias são confeccionadas logo no pós-operatório imediato, com a finalidade de evitar contaminações, infecções e hemorragias, além de facilitar a alimentação e deglutição. Tem também como vantagem a melhora na cicatrização, redução do tempo de uso de sonda, apoio curativo e auxílio na fala e recuperação psicológica do paciente (ARUN KUMAR VR, et al., 2016; SINGH M, et al., 2013). As próteses obturadoras permanentes são confeccionadas após a estabilização e cura, sendo menos invasivas e excluindo a necessidade de uma segunda cirurgia (LINGEN MW, 2016). No caso clínico apresentado, o paciente relatou uso prolongado de prótese obturadora provisória, esta foi construída com cobertura total do palato com acrílico e retenção com grampos interproximais de fio de aço ortodôntico. Este tipo de obturador deve ser utilizado apenas enquanto durar o pós-operatório, pois a longo prazo além de possível desadaptação após cicatrização, podem ocasionar lesões aos tecidos de suporte dos dentes pilares (ARUN KUMAR VR, et al., 2016). Inicialmente o paciente descartou a possibilidade de realizar novo procedimento cirúrgico, sendo então planejado um tratamento mais conservador com reabilitação protética definitiva por um obturador palatino sem dentes, visto que o paciente possuía todos os elementos em condições satisfatórias. Devido ao uso prolongado da prótese provisória, encontrou-se no exame inicial inflamações gengivais e acúmulo de biofilme evidente nos dentes do arco superior, o que exigiu a realização do preparo de boca prévio com raspagens e profilaxia. 
Segundo Esteves AJ, et al. (2016) a confecção da prótese obturadora deve ser criteriosa, para que consiga obter retenção, estabilidade e vedamento satisfatório. Dos Santos DM, et al. (2016) afirma que a prótese deve obedecer a alguns princípios básicos: rigidez, forças oclusais direcionadas ao longo eixo do dente e retenção. Para Waechter J, et al. (2017) deve-se respeitar as condições anatômicas e sistêmicas do paciente, pois o sucesso na confecção de uma prótese se dá conforme as técnicas clínicas e laboratoriais utilizadas, e, também, quanto ao material escolhido. Ottria L, et al. (2014) afirma que a retenção e a estabilidade da prótese podem ser afetadas por diversos fatores, como o tamanho da área afetada, controle muscular, tecido disponível, o nível de retenção promovida pelos dentes. Um obturador convencional usa vários grampos, e em alguns casos a inserção e remoção da prótese pode levar a uma rápida perda de retenção e estabilidade. Porém, Clen C, et al. (2015) afirma que quando as próteses são bem confeccionadas além delas terem uma retenção durável de qualidade, apoio e estabilidade, a distribuição da carga ajudam no suporte.

Portanto, no caso clínico exposto, realizou-se a confecção de uma prótese obturadora palatina definitiva, com grampos em dentes eleitos como pilares que auxiliam na retenção e estabilidade, sendo a adaptação de acrílico crucial para o sucesso da prótese. A construção se deu de forma análoga a uma prótese parcial removível, em que as etapas de planejamento ocorreram com utilização do delineador para determinação de eixo de inserção/remoção mais adequado para os dentes pilares selecionados. A seleção do conector maior em barra dupla, ocorreu a fim de distribuir de forma mais equânime as forças geradas pela mastigação, e, possibilitar a construção da porção obturadora posterior em acrílico. Além disto, executou-se a confecção dos nichos no preparo de boca também conforme o preconizado em reabilitações por próteses removíveis convencionais (JORGE JH, et al., 2006; KURIAKOSE EM, et al., 2019). A porção acrílica obturadora deve se acomodar de forma passiva aos tecidos, com o intuito de evitar a passagem de fluídos para cavidade nasal, e, dependendo do tamanho da deformidade pode-se utilizar obturador oco para diminuir o peso da peça (DOMINGUES JM, et al., 2016; WAECHTER J, et al., 2017). Para o paciente em questão não foi necessário obturador oco, pois além da deformidade ser pequena a prótese não incluía dentes em sua construção.

Sampaio LO, et al. (2018) descreveu um caso clínico onde foi possível observar reabilitação estética e funcional do paciente utilizando uma prótese obturadora. Tal sucesso também foi alcançado no caso clínico apresentado, onde a reabilitação bucal com prótese obturadora palatina devolveu ao paciente a autoestima por melhorar a fonética, mastigação e deglutição, além de permitir através da sua remoção a possibilidade de um exame clínico rápido do defeito, caso o paciente possa vir a ter uma recidiva. $O$ tratamento reabilitador com prótese obturadora foi satisfatório, vedando a comunicação buco-sinusal e restabelecendo adequada função bucal, promovendo maior conforto ao paciente, autoestima e qualidade de vida. O cirurgião-dentista exerce um papel importante no atendimento de pacientes oncológicos, ajudando significativamente a minimizar as sequelas ocasionadas pela doença e tratamentos.

\section{REFERÊNCIAS}

1. AMERICAN CANCER SOCIETY. Oral cavity and oropharyngeal cancer causes, risk factors, and prevention. Atlanta. 2005.

2. ARUN KUMAR VR, et al. Rehabilitation of maxillary defect by three different types of obturators - a cases series. J Pierre Fauchard Acad, 2016; 30(1): 1-5.

3. CHEN C, et al. Function of obturator prosthesis after maxillectomy and prosthetic obturator rehabilitation. Braz $\mathrm{J}$ Otorhinolaryngol, 2016; 82(2): 177-83.

4. DOMINGUES JM, et al. Palatal obturator prosthesis: case series. RGO, 2016; out-dez.; 64(4): 477-83.

5. DOS SANTOS DM, et al. Reabilitação com prótese obturadora após maxilectomia parcial: relato de caso. Revista Odontológica de Araçatuba, 2016; mai-ago.; 37(2): 52-66.

6. ESTEVES AJ, et al. Reabilitação protética de paciente oncológico: relato de caso. Arch Health Invest, 2016; dez.; $5(6): 291-2$.

7. GOIATO MC, et al. Fatores que levam à utilização de uma prótese obturadora. RGO, 2006; jul-dez.; 27(2): 101-6.

8. INTERNATIONAL AGENCY FOR RESEARCH ON CANCER. Global data on incidence of oral cancer. WHO. Geneva. 2012.

9. INSTITUTO NACIONAL DE CÂNCER. Estimativa 2018: incidência de câncer no Brasil. Rio de Janeiro. 2018.

REAOdonto | Vol. 1 | e3117| DOI: https://doi.org/10.25248/REAOdonto.e3117.2020 Página 7 de 8 
10. JORGE JH, et al. Preparos de dentes pilares para prótese parcial removível. Revista de Odontologia da UNESP, 2006; 35(3): 215-222.

11. KAR S e TRIPATHI A. Treatment outcome with delayed maxillary obturator prosthesis: case series of four patients. J Prosthodont, 2015; 25(2): 174-7.

12. KURIAKOSE EM, et al. Cast Partial Denture: A Preferred Choice of the Patient over Acrylic Partial and Flexible Denture-A Case Report, 2019; 5(3).

13. LINGEN MW. Cabeça e pescoço: glândulas salivares. In: Kumar V, Abbas AK, Aster JC. Patologia: bases patológicas das doenças. 9a ed. Rio de Janeiro: Elsevier; 2016; p. 751-71.

14. MATHEW AL, et al. Mucoepidermoid carcinoma of palate - a rare entily. Clinics and Practice, 2017; 7(4): 145-7.

15. OTTRIA L, et al. Maxilo-facial prosthesis (P.M.F): in a case of oral-nasal communication post-surgery an postradiotherapy. Oral \& Implantology, 2014; apr-jun.; 7(2): 46-50.

16. RABELO DP, et al. Reabilitação protética oral em paciente oncológico. Revista da Universidade Vale do Rio Verde, 2018; jan-jul.; 16(1): 1-8.

17. SAMPAIO LO, et al. Reabilitação protética em paciente com fissura palatina: relato de caso. Rev Uningá, 2018; outdez.; 55(S3): 128-36.

18. SHIBAYAMA R, et al. Reabilitação protética de paciente maxilectomizados - relato de caso. Revista Odontológica de Araçatuba, 2016; mai-ago.; 37(2): 9-16.

19. SINGH M, et al. Obturator prosthesis for hemimaxillectomy patients. Nalt J Maxillofac Surg, 2013; jan-jun.; 4(1): 11720.

20. WAECHTER J, et al. Oral and maxillofacial rehabilitation of a patient suffering from intraosseous adenoid cystic carcinoma. RGO, 2017; abr-jun.; 65(2): 168-73. 